Luc Prud'homme, Ph. D.

Codirecteur du LISIS

Professeur

Geneviève Bergeron, Ph. D.

Chercheure régulière au LISIS

Professeure

Annabelle Fortin, B. Ed.

Étudiante à la maîtrise en éducation Université du Québec à Trois-Rivières

\section{Apprendre à différencier : le sens construit par neuf enseignantes en formation initiale engagées dans une démarche d'étude de soi}

Learning to differentiate: Constructed meanings by nine teachers-in-training using a self-study approach

doi:10.18162/fp.2015.277

\section{ésumé}

En contexte de diversité, les enseignants sont incités à différencier. $0 r$, ce travail en classe demeure le fait de cas isolés, une majorité d'acteurs évoquant une formation initiale lacunaire pour y arriver. Notre projet visait à explorer le sens que peuvent attribuer des étudiants-stagiaires à la différenciation dans une méthodologie intégrant l'étude de soi comme approche prometteuse à cet effet. Les résultats démontrent que ce type de dispositif offre un contexte favorable à la construction d'une vision de l'enseignement plus ouverte à la diversité, légitimant le recours à des intentions et des actions variées au cours d'une situation pédagogique.

Mots-clés

Formation initiale, différenciation, étude de soi, diversité, enseignement, stagiaire, situation pédagogique, hétérogénéité

Abstract

In the context of diversity, teachers are encouraged to differentiate their teaching. However, there are, in fact, only isolated cases in the classroom. The majority of teachers evoke an incomplete initial training to be able to do so. Our project explores the meaning differentiation has to interns using a self study methodology as a promising approach for this purpose. The results show that this type of approach

provides a favorable context for the construction of a more open view of teaching to diversity which legitimizes the use of various intentions and actions during a teaching situation.

Keywords

Teacher education, educational differentiation, self study, diversity, teaching perspective, intern, general model of teaching

\section{Contexte de mutations sociétales}

De nombreuses mutations sociétales à l'heure actuelle, telles que la globalisation, le développement accéléré des sciences et technologies ainsi que la mobilité des populations, ont des incidences certaines sur le "vivre ensemble", interpellant des transformations au sein de nos institutions. Traitant plus spécifiquement de la diversité ethnoculturelle, religieuse et linguistique, McAndrew, Potvin et Borri-Anadon (2013) semblent indiquer que le contexte socio-environnemental contemporain ne peut plus occulter une réflexion autour d'institutions plus inclusives, que ce soit dans les sphères d'activités liées à la santé, à l'emploi ou à l'éducation. En ce sens, de par la nature des finalités qu'elle poursuit, l'école est l'une des institutions fortement interpellées par ce mouvement plus inclusif (Bouvier, 2012).

Ainsi, nombreux sont les écrits scientifiques qui convient les acteurs scolaires à repenser les manières d'enseigner considérées comme une norme depuis plus d'un siècle (Ainscow, 2009; Ducette, Sewell et Poliner Shapiro, 1996; Forlin, 2010). Plus encore, la recherche dans le champ de l'inclusion scolaire décrit le recours à une différenciation pédagogique comme une composante essentielle des pratiques en contexte de diversité (Armstrong, Armstrong et Spandagou, 2010; Booth et Ainscow, 2002; Mittler, 2005). Pour sa part, Darling-Hammond souligne dès 1996 que le défi en sciences de l'éducation est définitivement lié à l'intégration dans l'action de toutes les découvertes du $\mathrm{XX}^{\mathrm{e}}$ siècle au regard de l'apprentissage. Ce défi, tel qu'elle le formule, paraît interpeller une pratique de différenciation pédagogique. 
Si nous voulons vraiment que tous les élèves apprennent selon les nouveaux standards suggérés et les exigences complexes de la société contemporaine, nous devons développer des méthodes d'enseignement qui vont au-delà de la distribution d'information, de la passation d'examens et de l'attribution d'un résultat. Nous devons comprendre les méthodes d'enseignement qui répondent aux différentes manières d'apprendre des élèves, qui sont structurées de manière à tirer profit des points de départ spécifiques à chaque élève et, dans une logique d'étayage, qui soutiennent soigneusement le travail visant l'amélioration des performances. [Traduction libre] (1996, p. 7)

Sans référer explicitement à la différenciation, cette dernière évoque différentes manières d'apprendre et des points de départ variés qui semblent reliés à une reconnaissance explicite de la diversité que plusieurs associent à un travail de différenciation pédagogique (Tomlinson et al., 2003). Au-delà des problèmes liés à cette dénomination, il n'en demeure pas moins que la diversité dans un groupe scolaire existe, qu'elle peut être perçue à la fois comme un problème et comme une ressource et qu'elle s'associe à « l'expression de caractéristiques humaines ou de préférences de l'apprenant, faisant référence aux expériences déjà vécues qui sont interpellées alors qu'il aborde les situations nouvelles qui sont proposées en classe »(Prud'homme, 2007, cité dans Prud'homme, Vienneau, Ramel et Rousseau, 2011, p. 8). En ce sens, il demeure nécessaire de comprendre comment les acteurs scolaires peuvent composer avec le phénomène tout en ayant le sentiment du devoir accompli, une intention professionnelle qui ne peut se dissocier d'un certain travail de différenciation pédagogique (Tomlinson et McTighe, 2010).

\section{Problématique}

Or, nombreux sont les enseignants qui manifestent un sentiment d'incompétence face aux multiples défis qui sont associés à la diversité en classe (Bergeron, 2014; Horne et Timmons, 2009; Prud'homme, Samson, Lacelle et Marion, 2011). Il semble que les enseignants éprouvent toujours des difficultés à considérer la diversité en classe autrement qu'en termes de problèmes et demeurent campés dans une vision du travail plutôt transmissive et uniforme. Au Québec, Gaudreau et al. (2008) constatent que malgré les incitations à différentes formes de pratiques plus souples et plus flexibles pour répondre aux besoins de tous les élèves en classe dite ordinaire, l'enseignement demeure généralement assez conventionnel, privilégiant par le fait même certains modes de pensée et des stratégies plus analytiques d'appropriation de contenu. Ces résultats s'apparentent aux résultats de Pratt (2005) qui relève la dominance d'une perspective de transmission [Transmission perspective] chez les enseignants participant à sa recherche $(\mathrm{N}=253)$. Sans discréditer cette façon de concevoir le travail, ce dernier constate toutefois chez ces enseignants des difficultés certaines «à s'ajuster aux différences individuelles et à manifester une certaine empathie à l'égard des apprenants qui ne comprennent pas les contenus » [traduction libre] (p. 218). Or, il indique que l'apprentissage en contexte de diversité « [...] nous oblige à élargir nos horizons lorsqu'on réfléchit à la signification de l'enseignement » [Traduction libre] (Pratt, 2005, p. 4).

De surcroît, les auteurs du champ constatent que les dispositifs de formation initiale à l'enseignement demeurent lacunaires face aux enjeux de la diversité et de l'équité (Bergeron et St-Vincent, 2011; Forlin, 2010). C'est dans ce contexte que nous nous sommes intéressés à la problématique d'une formation pour mieux préparer les futurs enseignants à rencontrer la diversité en classe.

4 - Formation et profession 23(3), 2015 
D'emblée, tout en évoquant l'entrée imminente dans le XXIe siècle, Ducette et al. (1996, p. 369) maintiennent au terme d'une recension d'écrits portant sur la prise en compte de la diversité en éducation « [...] que le seul enseignant acceptable a une bonne connaissance de lui-même et un grand respect pour la diversité » [traduction libre]. Pour préciser cette conclusion, pouvant paraître quelque peu dogmatique, ils indiquent que le travail impliqué réclame des changements majeurs dans les programmes de formation initiale exigeant un travail sur la vision de l'enseignement qui oriente l'action de l'enseignant au regard de la diversité et de l'équité. Plusieurs chercheurs du champ de l'adaptation scolaire, de l'inclusion scolaire et de celui de la formation à l'enseignement abondent dans le même sens (Forlin, 2010; Gillig, 2006; Humphrey et al., 2006; Kane, Sandretto et Heath, 2002; LaBoskey, 2007; Tomlinson et al., 2003). Il s'agit de considérer la subjectivité et la rationalité de l'apprenant en formation pour favoriser des prises de conscience et des remises en question au regard d'un certain « curriculum caché » (Forlin, 2010). À cet effet, Kane et al. (2002) relèvent le caractère prometteur de l'étude de soi comme méthodologie de recherche pour agir autant sur le travail du formateur à l'enseignement que celui de l'enseignant. C'est dans cette perspective que nous avons précisé notre question générale de recherche : quel est le sens que peuvent construire des enseignants en formation initiale engagés dans une démarche d'étude de soi en regard de la différenciation pédagogique?

\section{Cadre conceptuel}

L'interdépendance entre le besoin d'apprendre à différencier en formation initiale et le caractère prometteur d'une étude de soi pour soutenir l'ouverture à la diversité sous-jacente à ce travail nous incite à définir dans un premier temps le sens que nous attribuons à la différenciation pédagogique au départ de la démarche de recherche. Dans un deuxième temps, nous clarifions celui d'un travail d'étude de soi préconisé pour favoriser le développement d'un engagement professionnel plus ouvert à la diversité.

\section{La différenciation pédagogique}

Considérant le caractère prolifique des publications sur la différenciation, nous avons d'abord tenté de délimiter un îlot de rationalité 1 autour du concept (Prud'homme et Bergeron, sous presse). Il s'agissait de dégager des écrits les attributs essentiels d'une différenciation pédagogique en tissant des liens entre son caractère polysémique [1], un dispositif de formation pour en soutenir l'apprentissage [2], et le projet contemporain de justice sociale et de réussite scolaire [3] dans lequel il est évoqué.

D'emblée, une compréhension de la diversité est sans contredit la prémisse à partir de laquelle le travail de conceptualisation de la différenciation doit s'effectuer (Rumelhard, 2002). Considérant les dangers de simplifications abusives, de stéréotypes et de visions à court terme qui peuvent s'associer à la reconnaissance de ce phénomène (Prud'homme, Vienneau et al., 2011), nous avons choisi de définir la diversité au fondement d'une différenciation pédagogique comme une manifestation à la fois dynamique et légitime de l'unicité de chacun des élèves. En ce sens, si la différenciation s'inscrit dans un projet professionnel d'ouverture à l'autre (projet d'inclusion et d'équité), de progression et de réussite (une lutte contre le déterminisme social et l'échec scolaire), un travail de conceptualisation implique le recours au principe d'éducabilité universelle, à la conviction qu'une manifestation de la diversité n'est 
jamais totalement statique et indépendante du contexte où elle se déploie (la situation pédagogique) et à une vision du caractère légitime de ce phénomène conçu comme un élément constitutif de l'équilibre d'un groupe.

Au départ de cette représentation de la diversité, la différenciation pédagogique peut se définir comme une façon de penser l'enseignement où l'enseignant conçoit des situations suffisamment flexibles pour permettre à tous les élèves de s'engager dans une tâche et progresser, tout en stimulant la création d'un climat d'interdépendance et d'intercompréhension entre les élèves qui permet de reconnaître la diversité en classe, de la valoriser et d'en tirer parti (Prud'homme, Leblanc, Paré, Fillion et Chapdelaine, 2015). Ainsi, un enseignant qui différencie cherche à articuler des situations collectives d'apprentissage qui permettent aux élèves de faire certains choix accentuant la signifiance du processus, tout en apportant aux besoins des adaptations et des modifications autant sur les contenus, les processus et les productions attendues. Une pratique de différenciation concerne donc à la fois [1] la conception des situations collectives d'apprentissage, [2] les ajustements (adaptations et modifications) pour répondre à certains besoins spécifiques et [3] les interventions liées à la gestion d'un environnement dans lequel les manifestations de la diversité sont légitimes (Tomlinson et al., 2003). Au départ de cette représentation, nous comprenons l'insistance de certains chercheurs (Kane et al., 2002) d'explorer une démarche d'étude de soi comme dispositif de formation pour favoriser le développement d'une posture d'ouverture à la diversité. Il s'agit d'un travail visant à rendre explicite (conscientiser) et à enrichir par la confrontation une vision professionnelle de l'enseignement plus favorable à l'expression de ce phénomène en classe.

Nos résultats indiquent l'importance d'amener les enseignants à réfléchir sur leur vision implicite de la classe et du curriculum de façon à apprécier le potentiel d'enrichissement que comporte la diversité. [Traduction libre] (Humphrey et al., 2006, p. 317)

\section{L'étude de soi}

L'étude de soi poursuit deux principaux objectifs que sont l'amélioration de l'enseignement et la découverte de savoirs liés à des questions éducatives. Les chercheurs engagés dans une démarche d'étude de soi visent à créer des produits tangibles et fonctionnels sous la forme de savoirs transférables aux collègues. Enracinée dans un courant épistémologique interprétatif et émancipateur, la méthodologie scientifique de l'étude de soi cherche donc à soutenir le travail du formateur de maître ou de l'enseignant qui aspire à améliorer sa pratique professionnelle (Loughran, Hamilton, LaBoskey et Russell, 2007). Cette approche considère l'apprentissage comme un processus qui tient compte des expériences, de l'histoire personnelle de vie et du contexte culturel. Ainsi, les chercheurs-formateurs du groupe SSTEP [Self-Study of Teacher Education Practices] créé lors du congrès annuel 1992 du American Educational Research Association démontrent l'importance d'amener les enseignants à comprendre et mettre au défi leurs théories implicites à propos de l'enseignement et d'eux-mêmes en tant qu'enseignants. Cette méthodologie offre à ces derniers des voies pour articuler et interroger leur histoire personnelle et les compréhensions résultantes. Cela permet également d'aller au-delà des routines et de chercher à répondre à de nouvelles questions (Louie, Drevdahl, Purdy et Stackman, 2003). Comme le décrit LaBoskey (2007), l'étude de soi est : 
[...] une méthodologie scientifique permettant d'étudier le contexte d'une pratique professionnelle [...]; elle est volontaire et centrée sur soi, comporte des visées d'amélioration, est interactive, inclut de multiples méthodes principalement qualitatives et définit la validité comme un processus constant de validation basé sur la véracité,l'authenticité et la confiance. [Traduction libre] (LaBoskey, 2007, p. 817).

L'étude de soi se comprend comme un mode d'enquête spécifique dans lequel les enseignants examinent les conflits entre leurs croyances et les actions à l'intérieur de leurs activités liées à l'enseignement dans la perspective d'examiner des questions pédagogiques (Whitehead, 1993). La réflexion critique est une composante essentielle de l'étude de soi et les auteurs du champ la voient comme le moyen d'investiguer une pratique et les croyances, intentions ou fondements qui l'orientent (Louie et al., 2003). Létude de soi implique des actes réflexifs individuels, mais surtout collectifs. En effet, elle est une démarche plus souvent collective et interactive. En fait, la construction des savoirs repose sur la confrontation des points de vue avec d'autres, ce qui implique la création d'une communauté d'apprenants (Laufgraben et Shapiro, 2004):-

L'étude de soi peut s'opérationnaliser à l'aide de la grande famille des recherches-actions où différentes formes de délibérations s'effectuent dans un processus de résolution de problème nourri par des données autant théoriques que pratiques, en lien avec l'objet de recherche. Globalement, il s'agit de réunir des étudiants qui acceptent volontairement de vivre une démarche de recherche participative et réflexive pour construire du sens autour d'une pratique de différenciation pédagogique.

\section{Méthodologie}

Au départ de ces considérations, nous avons choisi de créer un dispositif de formation interactive et réflexive se déroulant en parallèle au cursus obligatoire de formation initiale (cours, stages, séminaires). Il s'agissait de réunir des étudiants du programme de baccalauréat en enseignement préscolaire/ primaire (BEPEP) dans un projet de recherche-action-formation où différentes activités d'étude de soi accentuaient le sous-processus de formation inhérent à ce modèle de recherche (Dolbec et Prud'homme, 2009). La démarche proposait de coconstruire le sens que peut prendre la diversité en classe et une pratique de différenciation pédagogique. Afin de recruter les participants, le projet a d'abord été présenté à tous les étudiants ayant terminé une première année du programme. Au terme d'une rencontre d'information, neuf étudiantes inscrites en deuxième ou troisième du BEPEP manifestent leur intérêt et s'engagent dans la démarche d'une durée de deux années. Au moment du projet, elles sont toutes âgées de 20 à 25 ans et proviennent de différentes régions du Québec. L'objectif de recherche suivant oriente le travail des chercheurs-formateurs :

Décrire et analyser le sens de la différenciation tel que construit et conceptualisé dans l'expérience des enseignantes en formation initiale engagées dans une méthodologie de recherche accentuant une démarche d'étude de soi. 


\section{Les outils de collecte de données}

Trois outils de collectes de données sont exploités : les entretiens semi-dirigés individuels en amont et en aval de la démarche, les rencontres collectives et le journal de bord des participantes. Au total, neuf rencontres collectives se sont déroulées entre décembre 2009 et janvier 2012. Les entrevues et rencontres collectives ont été enregistrées et transcrites. Les données recueillies font l'objet d'une analyse qualitative interprétative thématique (Paillé et Mucchielli, 2008). Ce travail s'effectue inductivement au fur et à mesure que le projet évolue de manière à pouvoir soumettre les résultats préliminaires aux participantes et ainsi favoriser simultanément la coconstruction du savoir et une validation écologique. Les lignes qui suivent permettent d'illustrer plus spécifiquement comment outils et méthodes de collectes ont été utilisés et ajustés de manière à soutenir l'étude de soi et à recueillir des traces pertinentes du sens construit à travers le projet de recherche-action-formation.

\section{Description de la démarche}

Ce projet vise à soutenir la construction de sens en regard de la différenciation pédagogique (DP) de neuf étudiantes au BEPEP en stimulant un processus d'étude de soi. À cet effet, le travail des chercheurs prend différentes formes au cours du projet. Les entretiens semi-dirigés individuels effectués avant le début des rencontres collectives sont l'occasion pour les participantes de réfléchir et de se prononcer une première fois sur les thèmes centraux entourant l'objet d'étude : diversité, différenciation pédagogique, apprentissage et enseignement. Lors de la première rencontre collective, elles reçoivent d'ailleurs un compte-rendu de l'analyse de cet entretien. Elles sont invitées à prendre conscience des représentations se dégageant de leurs propos et à s'exprimer à cet effet. Les participantes ont ainsi l'occasion, dès cette première rencontre, de chercher à comprendre, de partager et de comparer leurs façons de voir.

De surcroit, lors de chacune des rencontres collectives, les participantes obtiennent un compte-rendu de la rencontre précédente. Celui-ci résume les principales discussions entourant les thèmes proposés, les points de concordance, de dissonance et comprend plusieurs citations des participantes. Encore une fois, elles sont invitées à prendre conscience de leurs représentations et à exprimer ce qu'elles comprennent de leur processus personnel et collectif de construction de sens. Cette stratégie stimule aussi les délibérations autour du sens qu'attribuent les autres participantes aux concepts et le travail d'introspection visant à favoriser les prises de conscience et remises en question entourant leurs croyances, valeurs et intentions respectives.

Des activités particulières sont planifiées pour soutenir simultanément l'étude de soi et la coconstruction de sens autour de la différenciation pédagogique : analyse de situations enseignement-apprentissage différenciées, visite des classes d'une école reconnue pour son orientation inclusive explicite, la lecture d'un texte simulant un trouble dyslexique, un travail sur l'analogie d'un enseignement monolithique (De Peretti, 1987), la construction de plans d'aménagement physique d'une classe multitâches, un questionnaire sur les perspectives d'enseignement (Pratt, 2005), l'analyse de la vidéo d'une classe inclusive, des discussions sur des faits divers de l'actualité en éducation et le travail d'anticipation sur les expérimentations possibles au cours des stages. 
Une part importante du travail des chercheurs lors des activités des rencontres collectives s'oriente autour de deux éléments : soutenir le dialogue cognitif et l'activité réflexive critique des participantes. Tout au long du processus, des efforts importants ont été déployés pour accorder une place prépondérante aux échanges, aux partages d'expérience et de points de vue. Globalement, le travail d'accompagnement et de planification des activités demeure très flexible et souple afin de s'ajuster aux besoins des participantes et de laisser place à ce qui émerge et fait sens pour elles.

La dernière rencontre collective du projet représente un moment crucial dans la collecte de données puisqu'elle vise à effectuer une synthèse du processus collectif vécu et du sens accordé à la DP. De plus, lors des entretiens semi-dirigés effectués en aval du projet, les participantes décrivent individuellement le sens final qu'elles accordent à la DP, leur processus de changement individuel (prises de conscience, remises en question, impacts du projet, activités ou apprentissages significatifs, etc.) ainsi que les forces et limites des différentes dimensions du projet de recherche-action-formation (étude de soi, discussion de groupe, activités, accompagnement, etc.).

Tout au long du projet, les participantes sont invitées à tenir un journal de bord. Elles sont libres d'y inscrire ce qui est significatif pour elles en relation avec les thèmes centraux du projet et en relation avec ce qu'elles découvrent en rapport à elles-mêmes. Ces éléments significatifs pouvaient découler des rencontres collectives du projet, mais aussi des expériences vécues dans le cadre des cours et des stages. Le journal de bord a été proposé dans la perspective de soutenir un processus réflexif critique continu et ainsi offrir des repères à l'étudiante pour effectuer l'entretien individuel final. Globalement, la construction de sens entourant le DP et l'étude de soi est à la fois une démarche individuelle et collective. Les participantes disposent de modalités pour progresser individuellement (journaux de bord et entretiens), mais les rencontres collectives soutiennent aussi les prises de conscience et remises en question de soi, ce qui ressort chez la totalité des participantes.

T'sais, tu te compares toujours aux autres, parce quen sachant comment toi t'es, ben tu vois tes différences avec les autres puis tu dis OK: elle, elle est comme ça. Puis, moi, je ne suis pas comme ça. T'sais, ça t'aide à plus te faire une image de toi-même! (Entretien en aval d'Anne-Sophie) / Tsé, je pense, c'est les autres qui mont aidée à me connaître! (Laurence) ${ }^{2}$

\section{Présentation des résultats}

Comme l'objet de cet article porte sur le sens construit par les participantes autour d'une différenciation pédagogique, nous décrivons dans cette section la vision construite autour de ce concept par les participantes. La démarche d'étude de soi effectuée pour y arriver fait l'objet d'une publication spécifique où nous illustrons que, malgré le caractère difficile et inconfortable de ce travail, l'étude de soi se révèle une composante incontournable d'un engagement professionnel pour mieux comprendre les choix pédagogiques d'une enseignante et élargir l'éventail d'options auxquelles elle peut recourir pour rencontrer la diversité en classe (Prud'homme et Bergeron, sous presse).

\section{Le sens construit autour de la différenciation pédagogique}

$\mathrm{Au}$ terme de la démarche de recherche, les participantes précisent le sens d'une différenciation pédagogique en évoquant des actions et des principes qui réfèrent à une vision de l'enseignement centrée sur les élèves et leurs besoins. 


\section{Une approche globale centrée sur les élèves}

D’emblée, elles conviennent qu'aujourd'hui, «enseigner, c'est différencier! C'est "différenseigner"! » (Gabrielle). Elles indiquent qu'il s'agit d'une façon de voir l'enseignement et l'éducation qui prend ses racines dans une analyse des particularités des élèves qui composent le groupe (leurs connaissances, leurs passions, leurs intérêts, leurs amitiés et leur famille). En arrivant en stage, nous dit l'une des participantes, «[...] la première chose que j'ai faite, c'est pas d'observer l'enseignante. J'observais les élèves! » (Alicia). Loin de considérer la différenciation comme une méthode, les participantes suggèrent qu'il s'agit davantage d'une " philosophie » ou d'une vision de l'enseignement centrée sur l'élève qui oriente le travail autour des différentes composantes d'une situation pédagogique.

Ben moi, la différenciation pédagogique, je vois ça comme une transversale. C'est pas une petite adaptation par-ci puis une petite adaptation par-là. [...] c'est un mode de vie de classe, un peu, si on veut. T'sais c'est une façon de voir le fonctionnement de ta classe, de voir la gestion de classe mélangée avec tes outils, mélangée avec ta façon d'enseigner, toutes les méthodes. (Alicia)

À plusieurs reprises, la différenciation est associée à une responsabilité pédagogique de s'assurer que tous les élèves dans un groupe sont en mesure d'apprendre en faisant une tâche scolaire. «[...] C'est comme de l'aide à l'apprentissage, [...] t'es pas obligé d'avoir un trouble [...] pour avoir besoin de différenciation! » précise Anne-Sophie tandis qu'Alicia donne des exemples tirés de son dernier stage en évoquant explicitement une certaine zone proximale de développement.

Le niveau de difficulté des textes que je leur offre est différent pour chacun de mes élèves. Ca aussi, pour moi, c'est de la différenciation, parce que [...], j’essaie de rester dans leur zone proximale!

\section{Une approche qui oriente les choix en situation pédagogique}

Afin de présenter certains des résultats de la recherche, le modèle de la situation pédagogique (voir figure 1) est repris pour illustrer l'orientation des actions que les participantes associent à la DP.

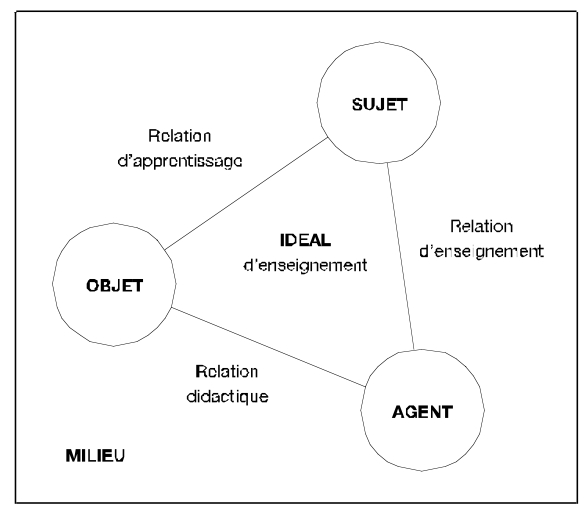

Figure 1

Modèle de la situation pédagogique (adapté de Legendre, 2005 et Pratt, 2005) 
D'entrée de jeu, elles suggèrent que l'agent [l'enseignant] qui veut différencier manifeste un engagement continu de réflexion et de remise en question sur lui en relation avec la diversité qui sera toujours présente dans un groupe.

En considérant les prises de conscience effectuées au cours de la démarche, largement associées à la connaissance de soi que stimulent les délibérations sur la diversité, elles suggèrent qu'un groupe d'élèves [les sujets] a tout avantage à apprendre à composer avec le phénomène en classe. Il s'agit pour elles de créer un climat de classe où les élèves sont invités à reconnaître ce qui les différencie les uns des autres, ce qui semble pouvoir favoriser une meilleure connaissance de soi pour chacun (ses forces, ses talents, ses façons de faire, de réagir, etc.). Il s'agit d'ailleurs de l'un des attributs essentiels pour soutenir notamment les élèves qui éprouvent des difficultés à l'école (Campbell-Whatley, 2008).

[...] il faut se connaître soi, mais il faut connaître nos élèves. Donc pour connaître nos élèves... les amener à se connaître, mais pas juste eux, mais entre eux [...] moi, je vois quion est une communauté d'apprentissage. [...] Il y en a, dans la classe, qui ont des forces. Ils doivent les connaître. Ils doivent s'en rendre compte, mais c'est pas nécessairement lui tout seul qui va s'en rendre compte. C'est peut-être les autres [qui vont l'aider à les voir]. (Émilie)

En ce sens, l'environnement physique et humain [le milieu] dans lequel les élèves et l'enseignant évoluent est associé à la construction d'un microcosme où les protagonistes cherchent à comprendre et s'intéressent à ce qui est différent de soi, une intention permettant aux apprenants d'élargir leur compréhension au contact de ce qui est moins familier. Le caractère interactif de la démarche d'étude de soi a suscité des prises de conscience sur le rapport à la diversité que les participantes pouvaient entretenir et une nécessité d'être plus curieuse à l'égard de ce qui de prime abord peut paraître étrange ou dérangeant.

Je pensais me connaître presque à l'extrême, et je me rends compte que pas tant que ça. [...] Je me pensais ouverte à la différence et je me rends compte que je suis fermée. Quand les personnes sont différentes de moi, je me ferme. Je mentoure des personnes qui sont comme moi! C'est une dure constatation. En troisième année de bacc., je commence ma formation, je sens que je recommence! (Gabrielle)

C'était des gens que je connaissais moins, de les entendre parler d'eux [...], de voir des nouvelles personnes, ben ça, ça m’aide à voir différemment! Après ça, de dire $O K$, il ne faut pas que je me fie à ce que je vois tout de suite! (Gabrielle)

Ces prises de conscience et la reconnaissance de la diversité comme une ressource potentielle dans un groupe semblent associer un travail d'éducation à la citoyenneté à une pratique de différenciation pédagogique, une relation rarement élaborée dans les écrits spécifiques sur la différenciation. En effet, les participantes constatent que reconnaître, valoriser et tirer parti de la diversité dans un groupe encourage l'entraide entre les élèves (une interdépendance) et une meilleure connaissance de soi et des autres (une intercompréhension). 
$\mathrm{La}$ "gestion démocratique des apprentissages » [...] suppose aussi et surtout qu'il [chaque élève] s'inquiète de et s'intéresse à la réussite ou aux difficultés de ses camarades. Il faut qu'il s'étonne de ce que son voisin n'apprenne pas aussi vite ou aussi facilement que lui, et que cet étonnement devienne pour lui un défi à relever; [...] par intérêt et passion. L'école préparera ainsi la transition entre la société laborieuse d'aujourd'hui et la société pédagogique de demain. (Galichet, 2001, p. 38)

Quant au contenu d'enseignement [les objets], la vision plus centrée sur les besoins des élèves paraît légitimer le fait que ces derniers doivent par-dessus tout être engagés dans une tâche où ils peuvent progresser par opposition à cette idée qu'ils doivent accomplir une tâche identique, ce qu'une participante illustre en racontant une expérimentation effectuée en stage.

[...] la différenciation, ça permet à tous les élèves de travailler là où ils sont rendus ou à peu près. Puisquils peuvent avancer un petit pas de plus. [...] Mais comme en lecture, ils ont appliqué différentes stratégies de lecture. Mes équipes, c'était pas les mêmes stratégies. Puis quand on a fait un retour tout le monde ensemble puis que les équipes expliquaient leurs stratégies, cétait pas les mêmes. Puis c'est normal, parce que leur niveau n'est pas le même. (Alicia)

En somme, la différenciation pédagogique se comprend par les participantes comme une vision de l'enseignement où un idéal d'interdépendance et d'intercompréhension oriente les prises de décisions à l'égard des relations d'apprentissage, d'enseignement et didactiques au cœur du travail de l'enseignant. Ce dernier reconnaît la légitimité d'une diversité de besoins, d'intérêts et d'expériences qui teinte l'engagement et la participation des élèves en classe. En ce sens, la compréhension d'un travail de différenciation peut s'articuler dans la mesure où il y a un engagement professionnel à reconnaître, à valoriser et à tirer parti des manifestations de la diversité qui se produisent en classe. Cet engagement peut se traduire par l'acceptation que tous les élèves ne peuvent pas nécessairement être à l'œuvre de manière constante sur les mêmes objets d'apprentissage et que leurs besoins soutiennent l'identification de ce qu'ils ont à travailler. Il implique l'exploitation de différentes stratégies d'enseignement et la reconnaissance de la légitimité de cette "palette de possibles" par tous les membres d'une classe, conçue davantage comme une communauté d'apprentissage. Il permet à l'enseignant, selon l'analyse des besoins qui se manifestent, de varier la nature du travail qu'il effectue pour aider un élève ou un groupe d'élèves à comprendre : médiation, facilitation, communication, guidage.

De manière plus pragmatique, les enseignantes mobilisées dans cette coconstruction de sens entourant la DP reconnaissent que différencier, c'est s'engager [1] à rompre avec cette idée d'un même travail pour tous en même temps, [2] à exploiter différentes approches sur une base régulière (l'enseignement magistral certes, mais aussi des pratiques telles que l'apprentissage coopératif, l'enseignement explicite, les approches par projet, etc.), [3] à alterner entre le collectif, le sous-groupe et l'individuel et [4] à prévoir des tâches suffisamment flexibles pour permettre aux élèves d'intégrer intérêts, forces ou talents dans leur production et ainsi augmenter la signifiance de la tâche. Il y a donc dans cette vision une ouverture à la diversité qui se traduit par l'exploitation de différentes actions pédagogiques plus éclectiques que dogmatiques. 
J'ai compris quion avait avantage à exploiter le groupe, les équipes et l'individuel. Il y a des élèves qui ont besoin de voir le contenu en groupe. Il y a d'autres qui ont besoin de le voir en sous-groupes puis de discuter, de confronter ce qu'ils ont appris. Puis il y a des élèves qui ont besoin de le faire tout seuls aussi. En [exploitant] ces trois étapes-là, je trouve que je vais chercher le maximum délèves. [...] (Alicia)

\section{Conclusion}

Globalement, le sens construit par les participantes reflète l'état d'avancement des connaissances déjà établies en matière de différenciation pédagogique tel que nous l'avons conceptualisé au départ de la démarche. Il est cependant intéressant de constater que les participantes traduisent cette vision de l'enseignement autant dans leur discours que dans des exemples d'expérimentations en stage. Tout en évoquant de multiples obstacles qu'elles anticipent dans l'actualisation de cette vision au cours $\mathrm{du}$ processus d'insertion professionnelle, elles reconnaissent la différenciation en termes d'option fondamentale de leur engagement dans la profession. De plus, l'analyse des résultats incite à poursuivre les investigations sur des relations, par ailleurs déjà pressenties (Perrenoud, 1997), qui existent entre une différenciation pédagogique et un certain travail d'éducation à la citoyenneté en classe. En effet, l'ouverture à la diversité s'associe explicitement à des intentions et des actions favorisant l'instauration d'un climat d'apprentissage où entraide, connaissance de soi et compréhension de l'autre sont valorisées dans la perspective de préparer les adultes de demain à mieux composer en société. En outre, les participantes reconnaissent que leur compréhension de la différenciation au terme de l'expérience est attribuable en grande partie aux bases de comparaison offertes par des exemples concrets et au travail interactif vécu pour favoriser une meilleure connaissance de soi. Car,

si je veux différencier, il faut que j'utilise l'outil le plus précieux que j’ai dans mes mains : moi! Pis, il faut que je le connaisse pour pouvoir l'utiliser! Tsé, si on veut utiliser un logiciel, il faut le connaître non? Ben, pour utiliser notre plein potentiel [...], il faut se connaître! (Gabrielle)

Dans ce sens, il demeure pertinent de poursuivre les investigations autour des démarches d'étude de soi pouvant s'articuler en formation initiale pour aider les étudiants à développer une vision de l'enseignement plus cohérente avec la réalité de l'hétérogénéité des classes. Le projet réunissait des participantes volontaires dans une démarche parallèle au dispositif formel, sans mesure d'évaluation et de certification. Elles reconnaissent que le dispositif offrait un contexte sécuritaire permettant progressivement d'être plus authentique, car «(...) dans un cours universitaire, on se met une espèce de filtre... Quion le veuille ou non, parce quion ne veut pas avoir l'air fou! » (Jo).

Or, ce type de dispositif en formation initiale semble offrir un contexte pour apprendre à différencier par le développement d'une vision de l'enseignement plus ouverte à la diversité. Il semble s'agir d'une « méthode d'enseignement » qui permet d'agir sur les valeurs, les croyances, les intentions et les actions des futurs enseignants pour s'engager à relever le défi évoqué par Darling-Hammond au regard de l'enseignement du XXI ${ }^{\mathrm{e}}$ siècle. 
Nous devons comprendre les méthodes d'enseignement qui répondent aux différentes manières d'apprendre des élèves, qui sont structurées de manière à tirer profit des points de départ spécifiques à chaque élève et, dans une logique d'étayage, qui soutiennent soigneusement le travail visant l'amélioration des performances. [Traduction libre] (DarlingHammond, 1996, p. 7).

\section{Remerciements}

Nous remercions d'abord les neuf participantes pour leur grande générosité tout au long de la démarche et le Fonds de recherche Société et culture du Québec du soutien financier obtenu pour la réalisation de ce projet de recherche.

\section{Notes}

1 En résumé, Fourez (1997, p. 218) décrit cette idée d'« îlot de rationalité » comme étant le « résultat d'un processus intellectuel » visant à créer une représentation théorique provisoire d'une situation précise pour ensuite pouvoir mieux en débattre.

2 Afin de préserver l'anonymat des participants, des pseudonymes sont utilisés pour toutes les citations illustrant leurs propos.

\section{Références}

Ainscow, M. (2009). Developing inclusive education systems : What are the levers for change? Dans P. Hick et G. Thomas (dir.), Inclusion and diversity in education: developing inclusive schools and school systems (vol. 2, p. 1-13). Londres, Royaume-Uni : Sage.

Armstrong, A. C., Armstrong, D. et Spandagou, I. (2010). Inclusive education : international policy et practice. Londres, Royaume-Uni : Sage.

Bergeron, G. (2014). Le développement de pratiques professionnelles inclusives : le cas d'une équipe-cycle de l'ordre d'enseignement secondaire engagée dans une recherche-action-formation (Thèse de doctorat, Université du Québec à Trois-Rivières, Canada). Repéré à http://www.archipel.uqam.ca/7022/1/D2730.pdf

Bergeron, G. et St-Vincent, L.-A. (2011). L'intégration scolaire au Québec : regard exploratoire sur les défis de la formation à l'enseignement au primaire et préscolaire. Éducation et francophonie, 39(2), 272-295. http://dx.doi.org/10.7202/ 1007738ar

Booth, T. et Ainscow, M. (2002). Index for inclusion : developing learning and participation in schools. Bristol, Royaume-Uni : Center for Studies on Inclusive Education.

Bouvier, A. (2012). Préparons l'École de 2030. Futuribles, (388), 51-72.

Campbell-Whatley, G. D. (2008). Teaching students about their disabilities : Increasing self-determination skills and self-concept. International Journal of Special Education, 23(2), 137-144. Repéré à http://files.eric.ed.gov/fulltext/ E]814451.pdf

Darling-Hammond, L. (1996). The right to learn and the advancement of teaching : Research, policy, and practice for democratic education. Educational Researcher, 25(6), 5-17. http://dx.doi.org/10.3102/0013189x025006005

De Peretti, A. (1987). Pour une école plurielle. Paris, France : Larousse.

Dolbec, A. et Prud'homme, L. (2009). La recherche-action. Dans B. Gauthier (dir.), Recherche sociale : De la problématique à la collecte de données (5éd., p. 531-569). Québec, QC : Presses de l’Université du Québec. 
Ducette, J. P., Sewell, T. E. et Poliner Shapiro, J. (1996). Diversity in education : Problems and possibilities. Dans F. B. Murray (dir.), The teacher educator's handbook : building a knowledge base for the preparation of teachers (p. 323-381). San Francisco, CA : Jossey-Bass.

Forlin, C. (2010). Reframing teacher education for inclusion. Dans C. Forlin (dir.), Teacher education for inclusion : Changing paradigms and innovative approaches (p. 3-12). New York, NY : Routledge.

Fourez, G. (1997). Qu’entendre par « îlot de rationalité »? Et par « îlot interdisciplinaire de rationalité »? Aster, 25, $217-225$. http://dx.doi.org/10.4267/2042/8686

Galichet, F. (2001). Quelle éducation à la citoyenneté dans une société défective? Dans M. Pagé, F. Ouellet et L. Cortesao (dir.), L'éducation à la citoyenneté (p. 27-39). Sherbrooke, QC : Éditions du CRP.

Gaudreau, L., Legault, F., Brodeur, M., Hurteau, M., Dunberry, A., Séguin, S. P. et Legendre, R. (2008). Rapport d'évaluation de la politique de l'adaptation scolaire. Repéré à http://www.education.gouv.qc.ca/fileadmin/site web/ documents/dpse/adaptation serv comp1/RapportEvalPolAdapScol.pdf

Gillig, J.-M. (2006). Intégrer l'enfant handicapé à l'école (3ééd.). Paris, France : Dunod.

Horne, P. E. et Timmons, V. (2009). Making it work : Teachers' perspectives on inclusion. International Journal of Inclusive Education, 13(3), 273-286. http://dx.doi.org/10.1080/13603110701433964

Humphrey, N., Bartolo, P., Ale, P., Calleja, C., Hofsaess, T., Janikova, V., ...Westo, G.-M. (2006). Understanding and responding to diversity in the primary classroom : An international study. European Journal of Teacher Education, 29(3), 305-318. http://dx.doi.org/10.1080/02619760600795122

Kane, R., Sandretto, S. et Heath, C. (2002). Telling half the story: A critical review of research on the teaching beliefs and practices of university academics. Revierw of Educational Research, 72(2), 177-228. http://dx.doi.org/10.3102/003465 $\underline{43072002177}$

LaBoskey, V.K. (2007). The methodology of self-study and its theoretical underpinnings. Dans J. J. Loughran, M. L. Hamilton, V. K. LaBoskey et T. Russell (dir.), International handbook of self-study of teaching and teacher education practices: Tome 2 (p. 817-869). Dordrecht, Pays-Bas : Springer.

Laufgraben, J. L. et Shapiro, N. S. (2004). Sustaining and improving learning communities. San Francisco, CA : Jossey-Bass.

Legendre, R. (2005). Dictionnaire actuel de l'éducation (3éd.). Montréal, QC : Guérin.

Loughran, J. J., Hamilton, M. L., LaBoskey, V. K. et Russell, T. (dir.). (2007). International handbook of self-study of teaching and teacher education practices: Tome 1 et 2. Dordrecht, Pays-Bas : Springer.

Louie, B.Y., Drevdahl, D. J., Purdy, J. M. et Stackman, R. W. (2003). Advancing the scholarship of teaching through collaborative self-study. Journal of Higher Education, 74(2), 150-171. http://dx.doi.org/10.1353/jhe.2003.0016

McAndrew, M., Potvin, M. et Borri-Anadon, C. (dir.). (2013). Le développement d'institutions inclusives en contexte de diversité : recherche, formation, partenariat. Québec, QC : Presses de l'Université du Québec.

Mittler, P. (2005). The global context of inclusive education : the role of the United Nations. Dans D. Mitchell (dir.), Contextualizing inclusive education : Evaluating old and new international perspectives (p. 22-36). Londres, RoyaumeUni : Routledge.

Paillé, P. et Mucchielli, A. (2008). L'analyse qualitative en sciences humaines et sociales (2 éd.). Paris, France : Armand Colin.

Perrenoud, P. (1997). Pédagogie différenciée : des intentions à l'action. Issy-les-Moulineaux, France : ESF.

Pratt, D. D. (2005). Five perspectives on teaching in adult and higher education. Malabar, FL : Krieger Publishing Company.

Prud'homme, L. et Bergeron, G. (sous presse). Diversité, étude de soi et engagement professionnel en formation initiale à l'enseignement. Dans F. Merhan, A. Jorro et J.-M. De Ketele (dir.), Mutations éducatives et engagement professionnel. Bruxelles, Belgique : De Boeck.

Prud'homme, L., Leblanc, M., Paré, M., Fillion, P.-L. et Chapdelaine, J. (2015). Différencier d'abord auprès de tous les élèves : un exemple en lecture. Québec Français, (174), 76-78. 
Prud'homme, L., Samson, G., Lacelle, N. et Marion, C. (2011). Apprendre à différencier au secondaire autour d'un objet transversal : la lecture. Les Dossiers des sciences de l'éducation, (26), 13-32.

Prud'homme, L., Vienneau, R., Ramel, S. et Rousseau, N. (2011). La légitimité de la diversité en éducation : réflexion sur l'inclusion. Éducation et francophonie, 39(2), 6-22. http://dx.doi.org/10.7202/1007725ar

Ruijs, N. M. et Peetsma T. D. (2009). Effects of inclusion on students with and without special educational needs reviewed. Educational Research Review, 4(2), 67-79. http://dx.doi.org/10.1016/j.edurev.2009.02.002

Rumelhard, G. (2002). Différences, singularité et universalité. Aster, 35, 17-37. http://dx.doi.org/10.4267/2042/8797

Savoie-Zajc, L. (2000). La recherche qualitative/interprétative en éducation. Dans T. Karsenti et L. Savoie-Zajc (dir.), Introduction à la recherche en éducation (p. 171-198). Sherbrooke, QC : Éditions du CRP.

Tomlinson, C. A., Brighton, C., Hertberg, H., Callahan, C. M., Moon, T. R., Brimijoin, K., .... Reynolds, T. (2003). Differentiating instruction in response to student readiness, interest, and learning profile in academically diverse classrooms : a review of literature. Journal for the Education of Gifted, 27(2-3), 119-145.http://dx.doi.org/10.1177/016 $\underline{235320302700203}$

Tomlinson, C. A. et McTighe, J. (2010). Intégrer la différenciation pédagogique et la planification à rebours. Montréal, QC : Chenelière éducation.

Whitehead, J. (1993). The growth of educational knowledge : Creating your own living educational theories. Bournemouth, Royaume-Uni : Hyde.

\section{Pour citer cet article}

Prud'homme, L., Bergeron, G. et Fortin, A. (2015). Apprendre à différencier : le sens construit par neuf enseignantes en formation initiale engagées dans une démarche d'étude de soi. Formation et profession, 23(3), 3-16.

http://dx.doi.org/10.18162/fp.2015.277 